Open Ocean • Here be Dragons 2018

\title{
Wheels of Poseidon Report
}

Daniel Oran ${ }^{1}$ Rachel Smith ${ }^{1}$ Dan Novy ${ }^{1}$ Siranush Babakhanova ${ }^{1}$ Brennan Phillips ${ }^{2}$ Allan Adams ${ }^{3}$ Chris Chronopoulos ${ }^{1}$

${ }^{1}$ MIT Media Lab, ${ }^{2}$ University of Rhode Island, ${ }^{3}$ MIT Future Ocean Lab

Published on: Oct 01, 2021

DOI: https://doi.org/10.21428/a680be9a.207c346e/56e2b8e0

License: Creative Commons Attribution 4.0 International License (CC-BY 4.0). 


\section{Challenge | Motivation}

Throughout the ages bioluminescence has inspired myths — and it's no wonder when massive blooms of jellyfish or algae can turn the ocean into a reflection of the night sky rife with stars from the depths. One such wonder has attracted the fascination of mankind for ages. Long ago, sailors in the Indian Ocean have long encountered massive bioluminescent blooms as they sailed through the water, lighting the wakes of their ships like the spokes of a wheel carrying them to their destination in a chariot of wind and water. They called this phenomenon "The Wheels of Poseidon.” Our goal was to harness the beauty of bioluminescence to create a new medium for artistic expression.

Specifically, we submitted the challenge of generating a living, programmable bioluminescent display, with pixels and voxels built of bioluminescent plankton floating freely in the water column and stimulated to glow by a programmable pattern of pressure waves in the water. Doing so required experts with a wide array of skills sets; through this collaboration we hope to have artistic, social, scientific, and technological impact.

\section{Goals}

Our goals were to:

1. Create a $1 \mathrm{D}$ prototype to assess the feasibility of a volumetric display

2. Create a $2 \mathrm{D}$ prototype

3. Create a 3D prototype

\section{Approach}

We built a 1D prototype by filling a 2" diameter clear pipe with fuciformis pyrocystis. The pipe contained a piezo transducer embedded in a resin impedance matched to water at one end. This Piezo was then driven by a small high voltage transformer hooked up to a signal generator. After significant testing, we found that the frequencies of sound with suitable wavelengths for a display (ie. $\sim 1 \mathrm{~cm}$ in water) killed the organisms before reaching an amplitude capable of creating bioluminescent standing waves. As such, it became clear that a volumetric display was not a viable option for a digital display.

Instead, we sought to use frequencies in a band that did not kill the dinoflagellates. To this end, we connected a transducer directly to the bottom of a large petri dish. This allowed us to generate a 2D display that relied on surface standing waves. As a result, we were able to create an inverse chladni plate where the dinoflagellates would light up at the crests of the standing wave patterns. The 2D display was successfully developed and demonstrated at the National Ocean Exploration Forum 2018: All Hands on Deck. 


\section{Next Steps}

Unfortunately the team around Wheels of Poseidon graduated so the project is unlikely to continue without a new champion. If things were to continue, we would scale up the 2D display and produce a music video that utilized the 2D standing wave display.

\section{Acknowledgements}

Wheels of Poseidon was funded by the MIT Media Lab Open Ocean Initiative. 